\title{
Designing Optimally Safe Robot Surface Properties for Minimizing the Stress Characteristics of Human-Robot Collisions
}

\author{
Jung-Jun Park, Sami Haddadin*, Jae-Bok Song, and Alin Albu-Schäffer
}

\begin{abstract}
Modeling of low severity soft-tissue injury due to unwanted collisions of a robot in collaborative settings is an important aspect to be treated in safe physical Human-Robot Interaction (pHRI). Up to now, safety evaluations for pHRI were mainly conducted by using safety criteria related with impact forces and head accelerations. These indicate severe injury in the robotics context and leave out low severity injury such as contusions and lacerations. However, for the design of an intrinsically safer robot arm, a reliable evaluation of the collision between a human and a robot that is based on skin injury criteria is essential. In this paper, we propose a novel human-robot collision model with and without covering, which is based on the impact stress distribution. The reliability of the proposed collision model is verified by a comparison with various cadaver experiments taken from existing biomechanical literature. Since the stress characteristics acting on the human head can be analyzed with this new collision model, the occurrence of certain soft-tissue injury can be estimated. Furthermore, the method serves for selecting the appropriate covering parameters, as e.g. elastic modulus and thickness, by evaluating the chosen skin injury indices.
\end{abstract}

\section{INTRODUCTION}

In the near future first service robots will work in human environments. Therefore, safety issues related to physical human-robot interaction (pHRI) have become increasingly important and considerable research efforts were conducted in this emerging field of robotics. Several researchers have devoted considerable effort to develop safer robot arms in order to guarantee human safety. Various collision experiments and safety estimations were conducted to quantify the potential danger of developed robot arms [1], [2], [3], [4], [5], [6].

The head is one of the most critical body parts to be protected from trauma because severe brain injury has of course devastating consequences. Thus, most researchers focused on severe head injury in order to evaluate the impact characteristics regarding injury between a human and a robot. Among several injury criteria used in car tests and in the standard biomechanical literature, the Head Injury Criterion (HIC) has been the most widely used in the robotics community [7], [8], [9], [10].

However, as no current service robot exceeds the safety critical thresholds of the HIC, it is not well suited to generate insight into low severity injury ${ }^{1}$. This is due to much lower collision speeds of nowadays robots compared to the ones in automobile crash-testing [11], [12]. Therefore, the fracture force of bones, e.g. facial and cranial bones, and the compression criterion for the chest were suggested to analyze more relevant injury mechanisms [11], [12]. Generally, the intrinsic blunt impact properties of collisions

The first two authors contributed equally to the work. J.-J. Park, S Haddadin*, and A. Albu-Schäffer are with the Institute of Robotics and Mechatronics, DLR - German Aerospace Center, Wessling, Germany, contact: sami.haddadinedlr. de

J.-B. Song is with the Intelligent Robotics Lab at Korea University, Seoul, Korea

${ }^{1}$ However, it is still well suited to state the range of injury in a coarser sense. between humans and robots were studied by applying various biomechanical severity indices [1], [2], leading to a basis of human injury analysis in robotics.

In the design of a service robot, not only life-threatening, but also slight injury and in the long term also human pain should be considered ${ }^{2}$. In this sense, [3] discussed the design of the minimum covering thickness of a robot arm required to prevent a collision force exceeding a criterion named Somatic Pain Tolerance of $50 \mathrm{~N}$. In [13], skin stress due to a blunt impact to a human body was analyzed for the evaluation of soft-tissue injury by considering the shape and material properties of a robot arm. To experimentally understand softtissue damage pig experiments for the analysis of stab and cut injuries were performed recently [14].

In this paper, we propose a novel collision model that is composed of a human head covered with a multi-layer structure and a robot arm with a soft covering. We validate this model against experimental cadaver data from the biomechanics literature and obtain the stress characteristics acting on the human skin. The model is based on the Hertz contact theory [15] and is verified with various experimental crashtest results from the biomechanical literature. The influence of robot parameters on the impact stress characteristics is used to choose a covering design that prevents soft-tissue injuries such as contusions and lacerations.

The approach to achieve this result consists of following steps. In order to predict lacerations and contusions, we need to analyze the stress distribution on the impact surface. For predicting this we developed a novel impact model. As the original blunt unpadded impact data in the biomechanics literature contains only impact forces and the according experimental conditions such as impact velocity, impactor curvature, and impactor mass, we still need to verify the proposed impact model. Then, for further comparison, we use existing padded impact experiments from literature to obtain covering material characteristics that are used in automobile crash-testing for passenger protection. Based on the developed model, we are now able to identify the influence of particular robot design parameters on the compressive and tensile stress, which are related to contusion and laceration tolerance of the human. Finally, we select the according robot covering material parameters that minimize both, maximum tensile stress and energy density without leading to a bulky design ${ }^{3}$.

The paper is organized as follows. Our collision model for physical human-robot impacts is proposed in Sec. II. Section III describes the influence of robot design parameters on impact stress. Various evaluations of soft-tissue injury due to the robot blunt impact and the determination of the design parameters of a robot for soft-tissue safety are provided in Sec. IV. Finally, we conclude in Sec. V.

\footnotetext{
${ }^{2}$ Please note that there is no direct relation between human pain and injury.

${ }^{3}$ The recent experimental verification of our model with crash-test dummies will be presented in a consecutive paper that we currently prepare.
} 


\section{Impact Model Between Robot And Human HeAd}

In this section we develop and validate the contact model for evaluating the impact stress during robot human collisions.

\section{A. Stress analysis between human and robot}

In this paper, we model the robot and the human head as two degrees-of-freedom (DoF) forced mass system moving along one common axis ${ }^{4}$. As depicted in Fig. 1, the robot is assumed to be a spherical mass, where $\nu, E, R$ and $m$ are the Poisson ratio, the elastic modulus, the radius and the reflected inertia of each material, respectively. Subscripts $r$, $c, s$ and $b$ represent the robot, the covering, the scalp and the skull bone. The Poisson ratio and the elastic modulus are material properties of the associated body part depending on its respective density. The sphere is covered with a second layer, which will later on represent the soft padding that is used to reduce the impact forces and stress. In general, the human head consists of multiple layers such as a scalp, skull, meninges, and the brain. To estimate stress induced skin injury, we only regard the skull that is fully covered by the scalp. Although diffuse injuries to the brain can be produced by high impact accelerations/forces, this is highly unlikely to occur during normal speed robot motion (up to $2 \mathrm{~m} / \mathrm{s}$ ), [10]. Therefore, we focus only on focal injuries such as contusions and lacerations that are directly caused by blunt impacts.
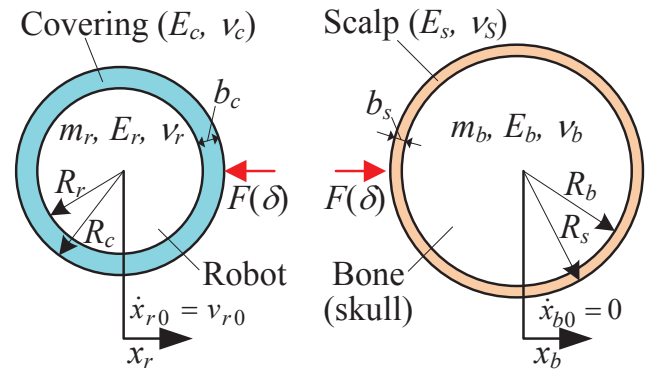

Fig. 1. Robot and human head model for the performed impact analysis.

During contact of the two masses the equation of motion in terms of the penetration depth $\delta=x_{r}-x_{b}$ is

$$
\ddot{\delta}=-\frac{F(\delta)}{m}, \quad \delta \geq 0
$$

where $F(\delta)$ and $m$ are the collision force and the effective mass, respectively. The effective mass can be expressed by $m=1 /\left(1 / m_{r}+1 / m_{b}\right)$.

Assuming homogeneous, isotropic, and frictionless bodies $^{5}$ as well as elastic deformations, Hertzian contact theory [15] can be applied. Furthermore, we assume that collision forces among all materials act according to the Hertz force law as a nonlinear elastic element. The collision force can be expressed as

$$
F(\delta)=\left\{\begin{array}{cc}
K_{c s} \delta^{n_{c s}} & \delta \leq b_{s m} \\
K_{c b}\left(\delta-b_{s m}\right)^{n_{c b}}+F\left(b_{s m}\right) & b_{s m}<\delta \leq b_{c m} \\
K_{r b}\left(\delta-b_{c m}\right)^{n_{r b}}+F\left(b_{c m}\right) & b_{c m}<\delta,
\end{array}\right.
$$

\footnotetext{
${ }^{4}$ During an impact the robot can be modeled by its respective reflected inertia and velocity in impact direction. Therefore, this model is sufficiently accurate if the correct configuration dependent parameters are used.

${ }^{5}$ This can be assumed to the fact that the radius of the contact area is assumed to be much smaller than the radius of the robot. This leads of course to very small tangential velocities.
}

where $K_{c s}, K_{c b}, K_{r b}$ are generalized stiffness constants between covering and scalp, covering and bone, and robot and bone, respectively. The exponents $n_{c s}, n_{c b}$ and $n_{r b}$ are determined depending on the contacting materials. For metallic materials, e.g. a value of 1.5 is used [15]. In this paper, exponents $n_{c s}, n_{c b}$ and $n_{r b}$ are set to $1.65,1.8$ and 2.65 , respectively ${ }^{6}$. The quantities $b_{s m}$ and $b_{c m}$ are maximum compression depth of the scalp and covering. When the penetration depth exceeds $80 \%$ of the thickness of a soft material such as covering and scalp, it is fully compressed $\left(b_{s m}=0.8 b_{s}, b_{c m}=0.8 b_{c}\right)$ [16]. Therefore, the collision force calculation has to switch at maximum scalp and covering compression accordingly.

The generalized stiffness constants (2) are

$$
\begin{aligned}
& K_{c s}=\frac{4}{3}\left(\frac{1-\nu_{c}^{2}}{E_{c}}+\frac{1-\nu_{s}^{2}}{E_{s}}\right)^{-1}\left(\frac{1}{R_{c}}+\frac{1}{R_{s}}\right)^{-\frac{1}{2}} \\
& K_{c b}=\frac{4}{3}\left(\frac{1-\nu_{c}^{2}}{E_{c}}+\frac{1-\nu_{b}^{2}}{E_{b}}\right)^{-1}\left(\frac{1}{R_{c}}+\frac{1}{R_{b}}\right)^{-\frac{1}{2}} \\
& K_{r b}=\frac{4}{3}\left(\frac{1-\nu_{r}^{2}}{E_{r}}+\frac{1-\nu_{b}^{2}}{E_{b}}\right)^{-1}\left(\frac{1}{R_{r}}+\frac{1}{R_{b}}\right)^{-\frac{1}{2}} .
\end{aligned}
$$

\section{B. Verification}

To verify the reliability of the collision model, we compare various simulation results with experimental cadaver data from existing biomechanical literature [17], [18], [19], [20], [21]. This cited research describes numerous blunt impact experiments with cadaver for six distinct experimental setups (see Fig. 2), thereby providing extensive experimental results that can be used for our analysis.
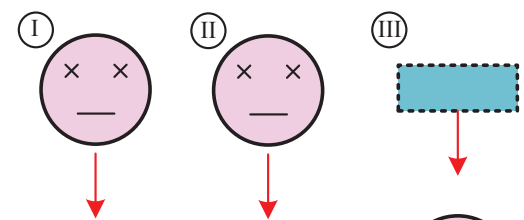

(IV)
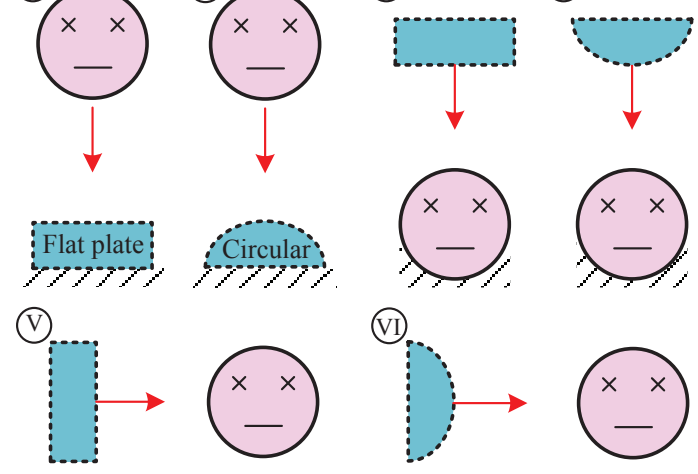

Fig. 2. Collision classes described in the biomechanical literature. (I) head drop-test to a fixed flat plate, (II) head drop-test to a fixed circular plate, (III) flat impactor drop-test to a fixed head, (IV) circular impactor drop-test to fixed head, (V) unconstrained collision between flat plate and head, and (VI) unconstrained collision between circular plate and head.

In order to generate a valid model for predicting impact stress and force, we first need to analyze the case of no covering such that the impact process is not only dominated by the covering characteristics. This would otherwise leave (2) the correct verification of head material parameters open as a wide range of values would provide a good fit for this analysis.

In this paper, the weight of the human head $m_{h}$, the elastic modulus of the human head bone $E_{b}$, the poison ratio $\nu_{b}$

\footnotetext{
${ }^{6}$ These values are obtained from an experimental crash-test dummy series, which results we will present in a consecutive paper.
} 
are set to $4.5 \mathrm{~kg}, 6.5 \mathrm{GPa}$ and 0.22 , respectively. The head includes the weight of meninges and brain is set to $4.5 \mathrm{~kg}$. The Poisson ratio $\nu_{s}$, the elastic modulus $E_{s}$, and the scalp thickness $b_{s}$ are set $0.42,16.7 \mathrm{MPa}$ and $3 \mathrm{~mm}$, respectively [22]. The Poisson ratio $\nu_{r}$ and the elastic modulus $E_{r}$ of the impactor are 0.3 and $70 \mathrm{GPa}$.

Figure 3 depicts the comparison between experimental and simulation results for various collision cases of Fig. 2. Table I lists the experimental conditions of the collision cases. In this study, the comparison for collision case V is omitted because to our knowledge there exists no experimental data for the unconstrained collision with a flat-plate impactor. For case VI, the experimental results are described in Fig. 3 (e) and (f). In Fig. 3 (b), (e) and (f) the boxes representes the fracture force and the collision duration (only this data is available).

TABLE I

EXPERIMENTAL CONDITIONS OF FIG. 3.

\begin{tabular}{cccccc}
\hline $\begin{array}{c}\text { No. of } \\
\text { Fig. }\end{array}$ & $\begin{array}{c}\text { No. of } \\
\text { case }\end{array}$ & $\begin{array}{c}\text { Impact } \\
\text { position }\end{array}$ & $\begin{array}{c}v_{r 0} \\
(\mathrm{~m} / \mathrm{s})\end{array}$ & $\begin{array}{c}m_{r} \\
(\mathrm{~kg})\end{array}$ & $\begin{array}{c}R_{r} \\
(\mathrm{~m})\end{array}$ \\
\hline Fig. 3(a) & I & Frontal cadaver [17] & 2.2 & Fixed & flat \\
Fig. 3(b) & II & Frontal cadaver [17] & 3.0 & Fixed & 0.08 \\
Fig. 3(c) & III & Temporo cadaver [20] & 4.3 & 12 & flat \\
Fig. 3(d) & IV & Temporo cadaver [20] & 2.7 & 10.6 & 0.03 \\
Fig. 3(e) & VI & Frontal cadaver [18] & 3.5 & 4.5 & 0.03 \\
Fig. 3(f) & VI & Frontal cadaver [18] & 3.5 & 4.5 & 0.008 \\
\hline
\end{tabular}

The force/time curves obtained from the simulation match those of the cadaver experiments very well before skull fracture occurs, see Fig. 3. In the experimental data the slope of the force/time curve changes abruptly after the fracture incident. Since our collision model assumes homogeneous material that satisfies Hooke's law, the maximum force would occur at the point of the maximum penetration without fracture. Therefore, the validity of the proposed collision model is verified by using the collision force profile and the collision duration before fracture.

Figure 4 depicts the simulation results for impacts to the frontal bone (the collision velocity, weight, and radius of the impactor are set according to Tab. II). Figure 4 (a) depicts the analysis for the flat impact surface and Fig. 4 (b) shows the result for the circular surface. Collision case III and IV cause significantly higher impact forces than the other cases. This is of course due to the constrained head.

TABLE II

ANALYSis CONDITIONS OF Fig. 4.

\begin{tabular}{cccccc}
\hline $\begin{array}{c}\text { No. of } \\
\text { Fig. }\end{array}$ & $\begin{array}{c}\text { No. of } \\
\text { case }\end{array}$ & $\begin{array}{c}\text { Impact } \\
\text { position }\end{array}$ & $\begin{array}{c}v_{r 0} \\
(\mathrm{~m} / \mathrm{s})\end{array}$ & $\begin{array}{c}m_{r} \\
(\mathrm{~kg})\end{array}$ & $\begin{array}{c}R_{r} \\
(\mathrm{~m})\end{array}$ \\
\hline \multirow{3}{*}{ Fig. 4 } & I & Frontal bone & 2.0 & Fixed & flat \\
& II & Frontal bone & 2.0 & Fixed & 0.02 \\
& IV, V V & Frontal bone & 2.0 & 10 & flat \\
& & & & & \\
\hline
\end{tabular}

If the curvature radius of the robot increases, so does collision force, while the impact duration again decreases, see Fig. 4 (c), (d) and (e). For the flat impactor, the curvature is set to $\infty$, while for the circular impactor, it is $0.02 \mathrm{~m}$, respectively. As intuition already indicates, the contact area is a less significant factor by means of impact force.

Next, we evaluate the validity of the model for impacts between a soft-covered robot and a human head by comparison with experimental results given in [21]. Table III lists
TABLE III

IDENTIFIED PARAMETERS OF FIG. 5.

\begin{tabular}{cccccc}
\hline $\begin{array}{c}\text { No. of } \\
\text { Experiment }\end{array}$ & $\begin{array}{c}m_{r} \\
(\mathrm{~kg})\end{array}$ & $\begin{array}{c}v_{r 0} \\
(\mathrm{~m} / \mathrm{s})\end{array}$ & $\begin{array}{c}R_{h} \\
(\mathrm{~m})\end{array}$ & $\begin{array}{c}E_{c} \\
(\mathrm{MPa})\end{array}$ & $\nu_{c}$ \\
\hline Ex. 15 & 5.31 & 6.29 & 0.077 & 70 & 0.25 \\
Ex. 17 & 5.30 & 7.33 & 0.079 & 130 & 0.25 \\
Ex. 19 & 5.31 & 7.05 & 0.075 & 70 & 0.25 \\
Ex. 26 & 5.31 & 4.63 & 0.078 & 100 & 0.25 \\
Ex. 27 & 5.18 & 3.80 & 0.078 & 30 & 0.25 \\
Ex. 31 & 5.31 & 7.05 & 0.078 & 100 & 0.25 \\
\hline
\end{tabular}

the used simulation parameters. Since the material properties of the used covering $\left(E_{c}, \nu_{c}\right)$ were not given in [21], we identified them based on the experimental data via optimizing collision force and impact duration. The results for a set of analyzed experiments are listed in Tab. III. The parameters of the human model are set to the same values as for the rigid collision analysis above (without covering). For identification of the covering material parameters, we set $b_{c}=0.02 \mathrm{~m}$. This is enough to prevent the material from being fully compressed. Furthermore, the radius $R_{r}$ of the impactor is set to $0.01 \mathrm{~m}$. As the true radius is not known we chose the same value that was used in an impact study [19] that cited the original work [21] by the same authors.

Figure 5 depicts the comparison of the experiment with simulation. Both, the maximum collision force and collision duration show good agreement with the experimental results. Also the force shape is very similar. However, please note that the collision model is not able to reproduce the energy dissipation of the process.

To sum up, we verified the proposed impact model by systematically comparing it to padded and unpadded cadaver experiments. This enabled us to identify the appropriate material parameters of the involved bodies.

\section{INFLUENCE OF ROBOT DESIGN PARAMETERS}

In this section we derive the maximum stress acting on the human head from the dynamic differential equation of motion of the proposed collision model. We analyze how stress in influenced by the most important robot design parameters. The compressive stress applied to the human head due to a blunt impact is given by

$$
\sigma_{c}(\delta)=\left\{\begin{array}{cc}
\frac{3 F(\delta)}{2 \pi \delta}\left(\frac{1}{R_{c}}+\frac{1}{R_{s}}\right) & \delta \leq b_{s m} \\
\frac{3 F\left(\delta-b_{s m}\right)}{2 \pi\left(\delta-b_{s m}\right)}\left(\frac{1}{R_{c}}+\frac{1}{R_{b}}\right)+\sigma_{c}\left(\delta_{s m}\right) & b_{s m}<\delta \leq b_{c m} \\
\frac{3 F\left(\delta-b_{c m}\right)}{2 \pi\left(\delta-b_{c m}\right)}\left(\frac{1}{R_{r}}+\frac{1}{R_{b}}\right)+\sigma_{c}\left(\delta_{c m}\right) & b_{c m}<\delta,
\end{array}\right.
$$

where the compressive stress on the human head has its maximum value at $\delta=\delta_{\max }$.

\section{A. Influence of rigid robot arm parameters}

Typical design parameters of a robot arm are the masses, moments of inertia, the radius of curvature of the robot hull, and the maximum operating velocity. The stress characteristics acting on the human head can be calculated from (4). To obtain the relationship between stress and robot parameters, no covering is used.

Figure 6 shows the dependency of the maximum compressive stress on the head with respect to the reflected inertia of the robot and parameterized by impact velocities ranging from $0.5 \mathrm{~m} / \mathrm{s}$ to $3 \mathrm{~m} / \mathrm{s}$. The curvature radius of the robot is 


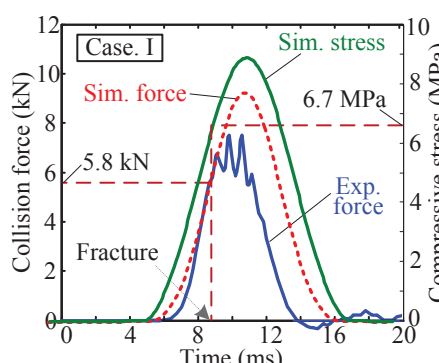

(a)

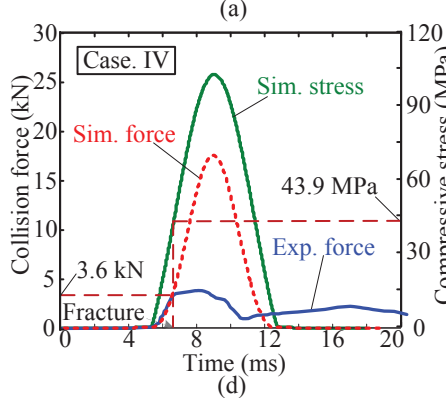

(d)

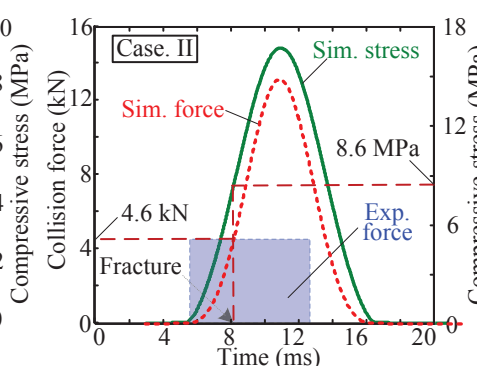

(b)

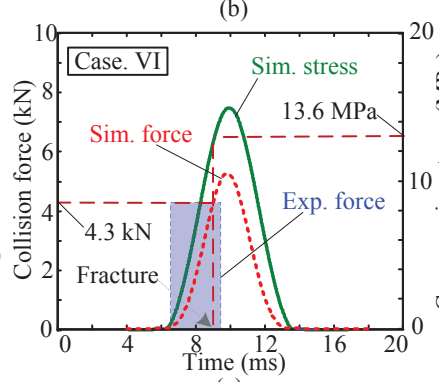

(e)
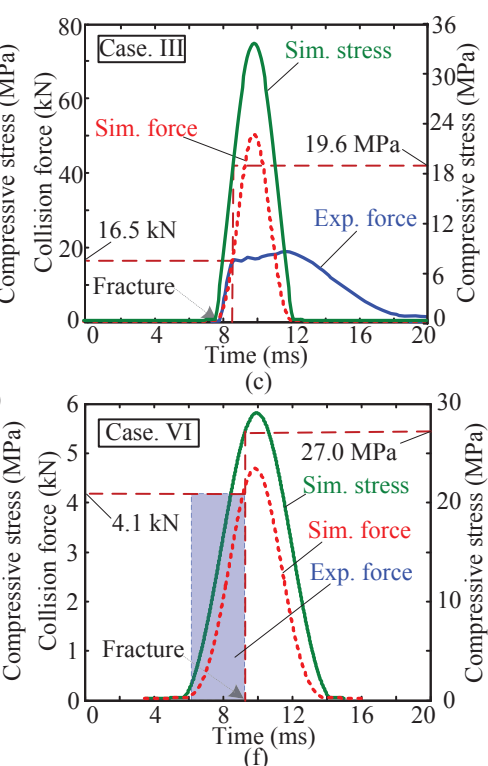

Fig. 3. Comparison between simulation and experimental results for various collision cases, where Sim. and Exp. represent simulation and experiment, respectively. The robot radius is chosen to be $R_{r}=0.01 \mathrm{~m}$.

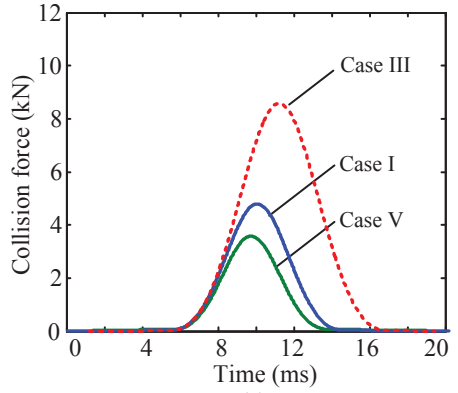

(a)

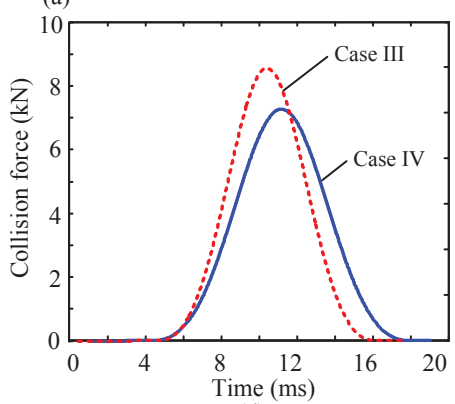

(d)

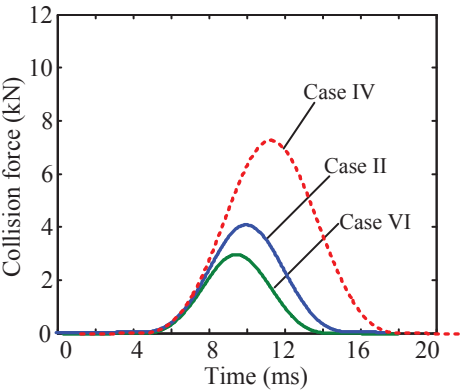

(b)

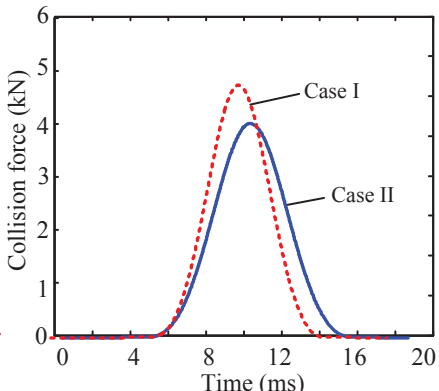

(c)

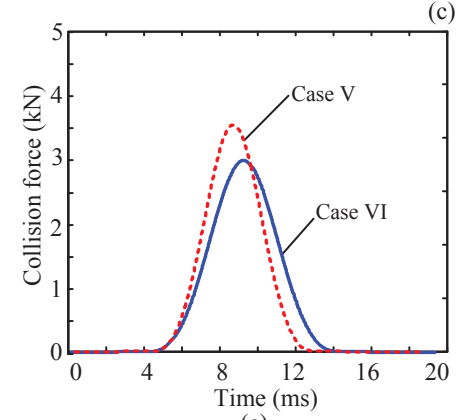

(e)

Fig. 4. Collision simulation results according to various collision cases. (a) flat impactor, (b) circular impactor, (c) head drop to constrained impactor, (d) impactor drop to constrained head, (e) collision between unconstrained head and impactor.

$0.05 \mathrm{~m}$. Its reflected inertia is obtained via the Jacobian and inertia matrix [23]. Clearly, Fig. 6 shows that the maximum compressive stress saturates for increasing reflected inertia at constant impact velocity. From a certain point on it therefore does not result in higher impact stress anymore.

Figure 7 depicts the maximum compressive stress as a function of the curvature radius and is parameterized by impact velocities ranging from $0.5 \mathrm{~m} / \mathrm{s}$ to $3 \mathrm{~m} / \mathrm{s}$. The reflected inertia of the robot is set to $10 \mathrm{~kg}$. The maximum stress reduces gradually with increasing curvature, however, only limited dependency on the radius can be confirmed for curvatures $\geq 0.1 \mathrm{~m}^{7}$. From these results it follows that the operating velocity is the more dominant factor of maximum stress for a typical industrial robot $\left(m_{r}>10 \mathrm{~kg}, R_{r}>0.05\right.$ $\mathrm{m})$ or the DLR Lightweight Robot III with a $6 \mathrm{~kg}$ payload $\left(m_{r}=4+6 \mathrm{~kg}, R_{r}=0.08 \mathrm{~m}\right)$.

\section{B. Influence of soft covering parameters}

Design parameters for the soft covering are the elastic modulus, the Poisson ratio, and the thickness. For the robot we assumed a small scaled industrial robot $\left(m_{r}=10 \mathrm{~kg}\right.$,

${ }^{7}$ Please note that (4) is only valid for radii that are larger than the contact area, i.e. non-sharp contact. 


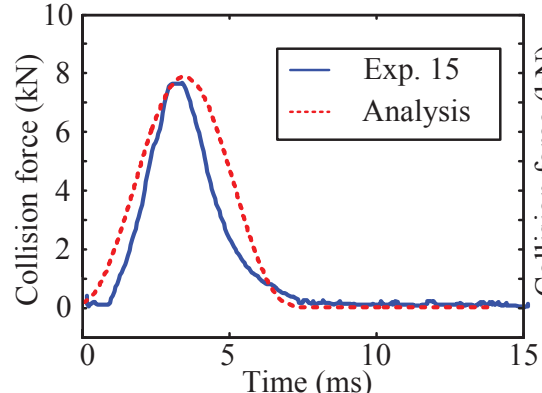

(a)

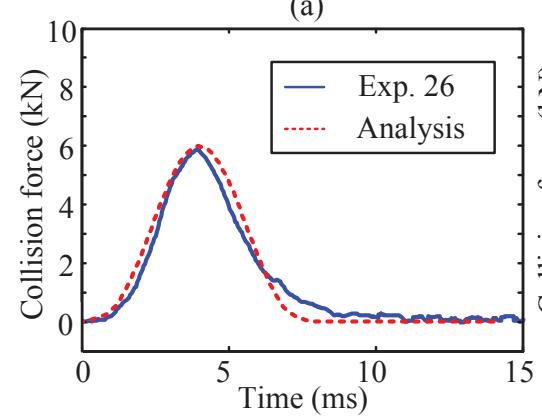

(d)

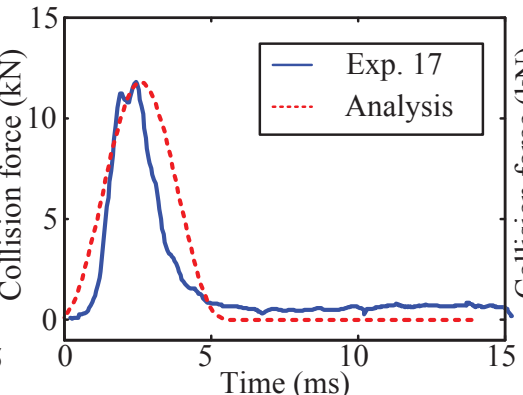

(b)

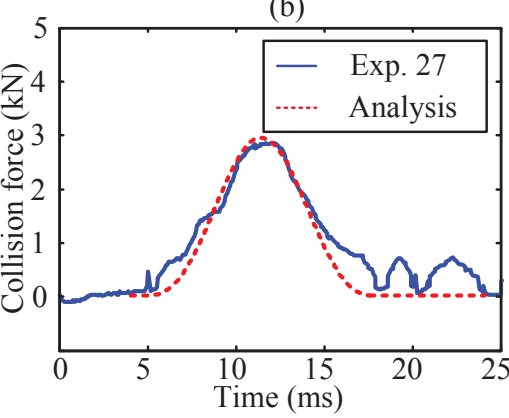

(e)

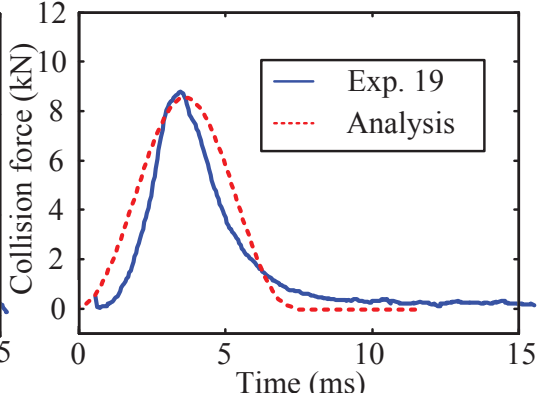

(c)

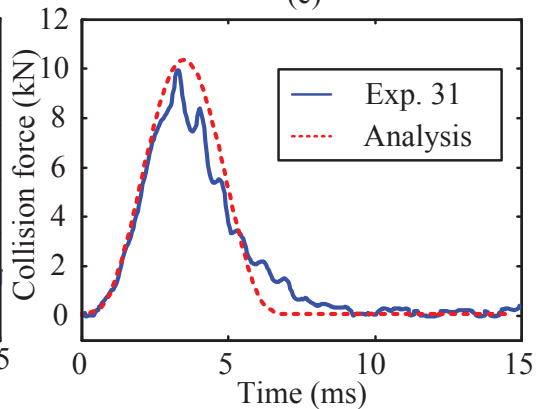

(f)

Fig. 5. Comparison between simulation and experimental results of robot impact to frontal bone with covering.

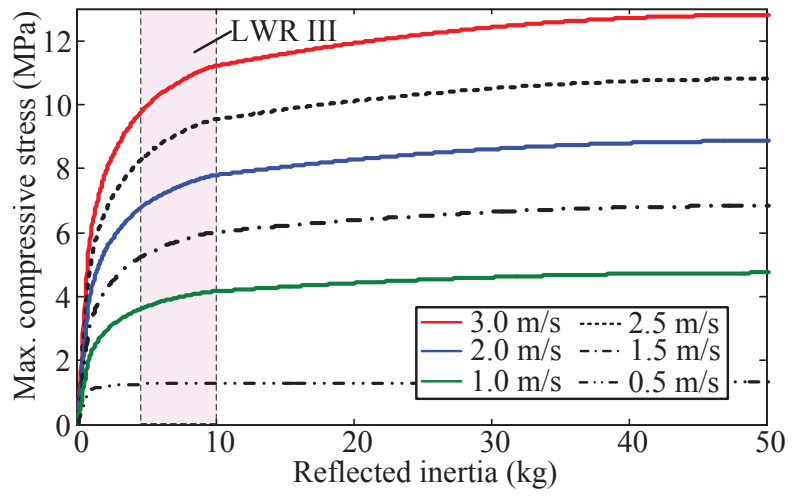

Fig. 6. Resulting maximum stress as function of collision velocity and reflected inertia for $R_{r}=0.05 \mathrm{~m}$ and no covering.

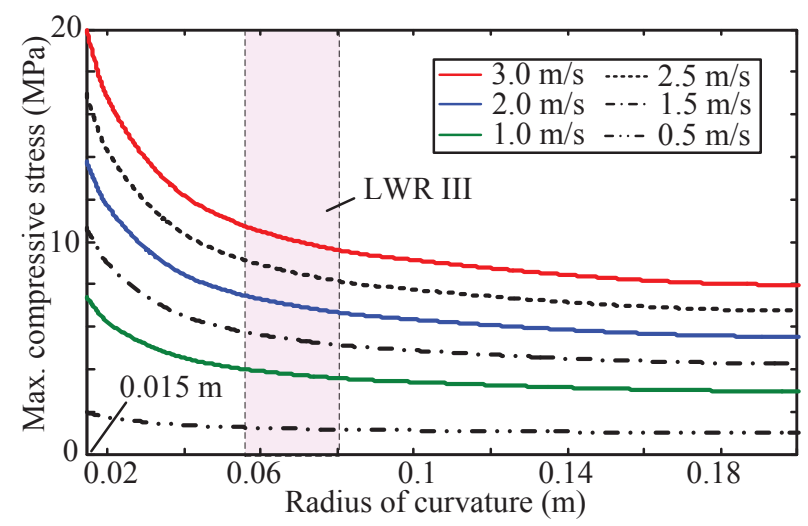

Fig. 7. Resulting maximum stress as a function of collision velocity and curvature radius for $m_{r}=10 \mathrm{~kg}$ and no covering. $\left.v_{r, 0}=2 \mathrm{~m} / \mathrm{s}, R_{r}=0.015 \mathrm{~m}\right)$. Figure 8 shows the relationship between maximum compressive stress acting on the head and the Poisson ratio parameterized by the covering elastic modulus for $b_{c}=20 \mathrm{~mm}$. This thickness prevents penetration depths that exceed the maximum compression depth of a covering $\nu_{c}$ that ranges from 0 to 0.25 . This corresponds to typical soft polymer foams. As one can see, the Poisson ratio has only marginal effect on the maximum compressive stress. The elastic modulus, on the other hand, significantly changes the curve characteristics.

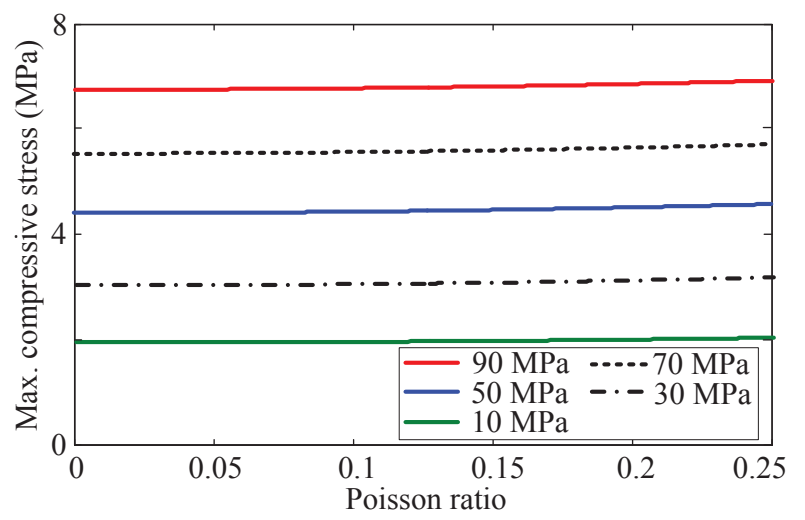

Fig. 8. Resulting maximum stress as a function of covering elastic modulus and Poisson ratio for $b_{c}=0.02 \mathrm{~m}, m_{r}=10 \mathrm{~kg}, v_{r, 0}=2 \mathrm{~m} / \mathrm{s}, R_{r}=$ $0.015 \mathrm{~m}$. The covering is not fully compressed.

Figure 9 denotes the maximum stress as a function of cover thickness and elastic modulus for $\nu_{c}=0.25$. It rapidly changes below a certain thickness, which is of course due to its full compression. Before this threshold thickness, the influence of increasing elastic modulus is immanent. However, also the effect of enlarging the elastic modulus saturates. For zero covering thickness the maximum stress converges 
for each thickness to the same value of $\approx 12.5 \mathrm{MPa}$. To sum up, the dominant design factors of a covering are the elastic modulus and its thickness. For the analyzed padding no exceedance of the fracture tolerance is observed.

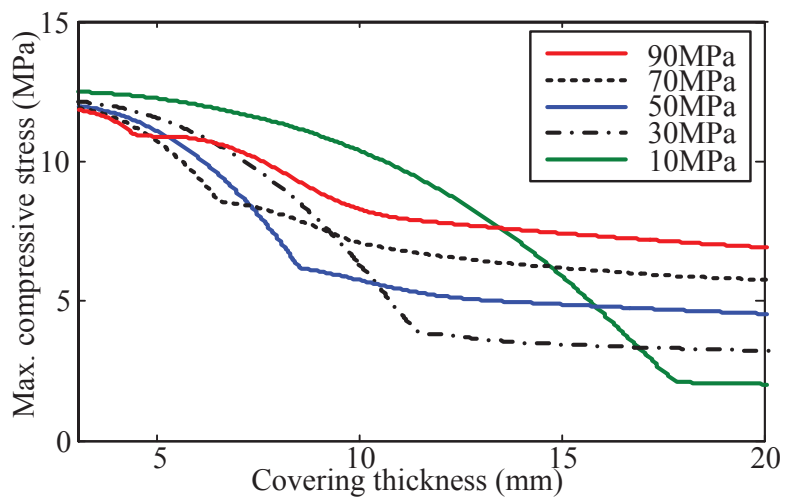

Fig. 9. Resulting maximum compressive stress as a function of elastic modulus and covering thickness for $\nu_{c}=0.25, R_{r}=0.015 \mathrm{~m}, v_{r, 0}=$ $2 \mathrm{~m} / \mathrm{s}$.

\section{DESIGN OF ROBOT COVERING}

In this section, we introduce skin injuries and estimate their magnitude by means of the introduced covering design parameters. For this we need to evaluate both compressive and shear stress as they indicate contusion and laceration, respectively.

\section{A. Safety criterion for soft-tissue injury} [24].

Soft-tissue injury can be generally classified as follows

- Abrasions or excoriations: an ablation of parts or the entire epidermis from the corium.

- Contusions or bruises: a type of relatively minor hematoma of tissue in which capillaries are damaged by trauma, allowing blood to seep into the surrounding interstitial tissues.

- Laceration: a tear in the tissue and irregular cuts caused by a blunt impact to soft tissue which lies over hard tissue.It is wider than deep.

- Puncture or stab: a break or opening wound in the skin caused by a clean sharp edge, which is usually characterized by being deeper than wide.

In this paper, we focus on laceration and contusion. Contusions are a matter of impact energy density $e_{A}$ [24]. However, please note that the injury tolerance of haematoma and suffusion are below this value. Lacerations occur usually where thin soft tissue layers are directly located above hard tissue material as e.g. the scalp. Application of tensile stress, which is larger than the ultimate tensile stress of skin potentially leads to skin fracture. In this paper, we use the ultimate tensile stress of the skin, which is assumed to be $1 \mathrm{MPa}$ [25], and the maximum energy density of $2.52 \mathrm{~J} / \mathrm{cm}^{2}$ as the injury thresholds for laceration and contusion, respectively.

\section{B. Selection of robot covering}

For estimating the aforementioned skin injury types, we need to calculate the tensile stress and the energy density. At the surface within contact area, the stress components are all compressive by nature, except at the very edge of contact where the radial stress is tensile as shown in Fig. 10, [15].

The tensile stress of the contacting material is obtained by

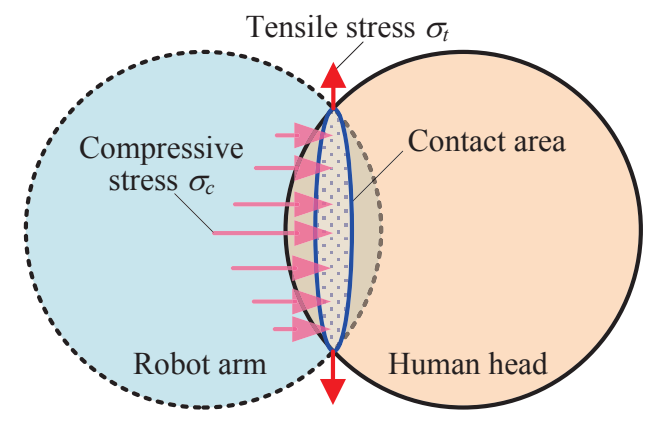

Fig. 10. Tensile stress due to compressive stress acting on the human head.

$$
\sigma_{t, \max }=\frac{\sigma_{c, \max }(1-2 \nu)}{3},
$$

where $\nu$ is the tensile Poisson ratio of the scalp, which is set to 0.42 . For estimating contusions the energy density can be expressed as follows.

$$
e_{A, \max }=\int_{0}^{\delta_{\max }} \sigma_{c}(\delta) \mathrm{d} \delta
$$

For our analysis, we assume $m_{r}=10 \mathrm{~kg}, v_{r 0}=2 \mathrm{~m} / \mathrm{s}$, and $R_{r}=0.015 \mathrm{~m}$ or $R_{r}=0.08 \mathrm{~m}$ (Again a small-scale industrial robot or the LWR-III with $6 \mathrm{~kg}$ payload). We choose $R_{r}=0.015 \mathrm{~m}$ as a worst-case according to Fig. 7 and $R_{r}=0.08$ as a rather moderate value.

Figure 11, 12 depict the results for the maximum tensile stress and the maximum energy density as a function of the elastic modulus and the thickness of a covering, respectively. Since the maximum density energy plot is very similar to the maximum tensile stress, both have their global minimum for similar conditions $\left(E_{c}\right.$ and $\left.b_{c}\right)$. According to Fig. 11, 12, contusions occur before the tensile stress on the skin exceeds the laceration tolerance value. This can be interpreted such that lacerations are accompanied by contusions of the skin.

In general, the tensile stress and the energy density decrease with increasing thickness. For preventing contusion the thickness and the elastic modulus of a covering should be larger than $15 \mathrm{~mm}$ and near $27 \mathrm{MPa}$, respectively .

Figure 13 shows the maximum tensile stress and the maximum energy density as a function of the elastic modulus for a thickness of $10 \mathrm{~mm}$ (upper) and $15 \mathrm{~mm}$ (lower) (cross sections of Fig. 11, 12). For the thinner solution lacerations can be prevented, however, contusions are still possible even for the optimally chosen elasticity modulus. For $b_{c}=14 \mathrm{~mm}$ both injuries can be fully prevented for an optimal material. As both energy density and tensile stress are minimized close to $E_{c}=27 \mathrm{MPa}$, polystyrene foam (No. $1, E_{c}=27.6 \mathrm{MPa}$ ) could be selected as an appropriate covering to guarantee skin safety, see Tab. IV.

TABLE IV

Properties of COVERING MATERIALS IN FIG. 12.

\begin{tabular}{ccccc}
\hline No. in Fig. 14 & Material & $E_{c}(\mathrm{MPa})$ & $\nu_{c}$ & Density $\left(\mathrm{kg} / \mathrm{cm}^{2}\right)$ \\
\hline 1 & Polystyrene & 27.6 & 0.22 & 50 \\
2 & Polystyrene & 48.3 & 0.25 & 100 \\
3 & Polyurethane & 62.1 & 0.20 & 100 \\
4 & Polyurethane & 137.9 & 0.29 & 200 \\
\hline
\end{tabular}




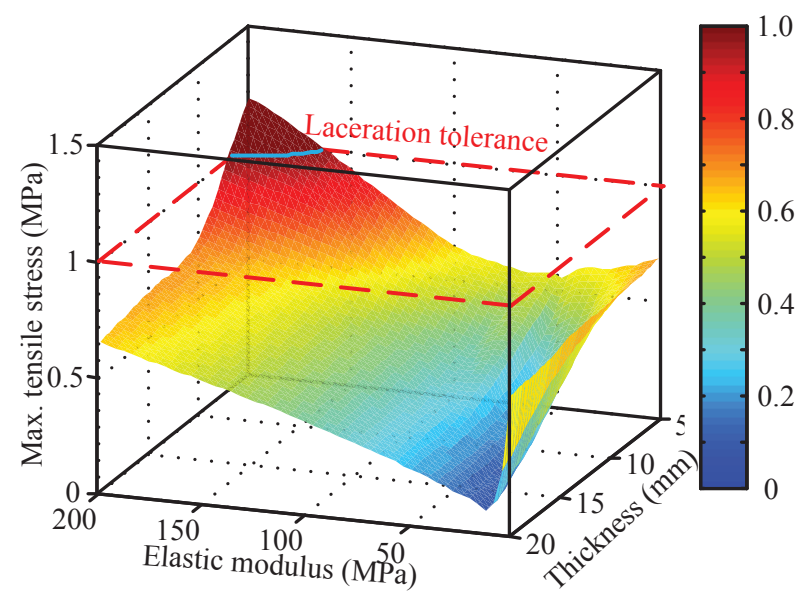

(a)

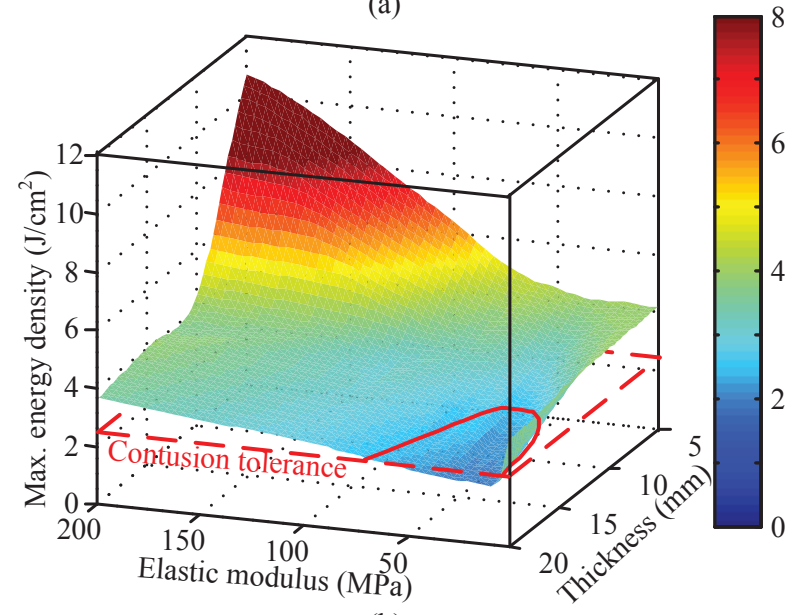

(b)

Fig. 11. Impact simulation for the head (frontal). (a) Max. tensile stress, and (b) max. energy density as a function of the elastic modulus and the thickness of covering for DLR LWR III with load. The impact speed is $v_{r, 0}=2 \mathrm{~m} / \mathrm{s}$ and the radius of the robot is $R_{r}=0.015 \mathrm{~m}$.

Figure 14 depicts the tensile stress/displacement curves of three different coverings with thickness $10 \mathrm{~mm}$ (upper) and $15 \mathrm{~mm}$ (lower). For compliant covering $\left(E_{c}<10 \mathrm{MPa}\right)$, the tensile stress maintains very low value until the penetration depth $\delta$ reaches the maximum compression. When the covering is fully compressed, the tensile stress abruptly increases. For the stiff covering $\left(E_{c}>150 \mathrm{MPa}\right)$, even though the covering is not fully compressed, the maximum tensile stress already reaches very high values. Finally, for the polystyrene foam $\left(E_{c}=28 \mathrm{MPa}\right)$ the tensile stress is significantly lower, as the aborbed energy is fully absorbed by the well designed covering without causing too high impact forces due to material property or full compression and contact with the robot.

\section{CONCLUSIONS}

In this paper, we proposed a novel collision model for analyzing laceration and contusion injury, which is used to design an optimally safe soft covering. We draw following conclusions:

1) The reliability of the proposed collision model was verified by comparing our simulation results with various experimental data of cadaver experiments from the biomechanical literature. This leads to a reliable analysis of blunt

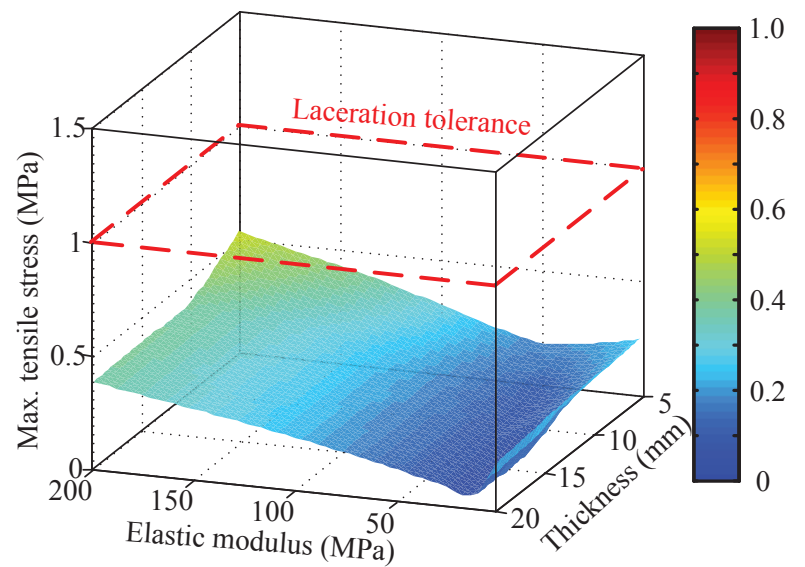

(a)

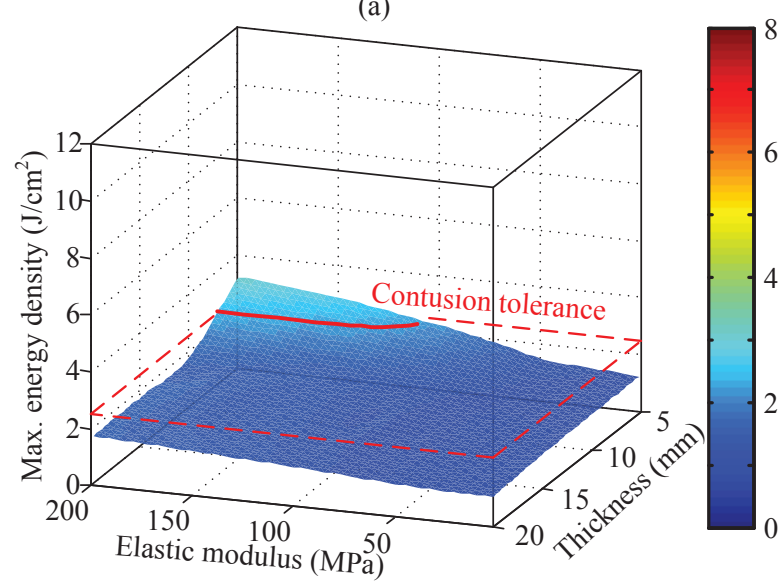

(b)

Fig. 12. Impact simulation for the head (frontal). (a) Max. tensile stress, and (b) max. energy density as a function of the elastic modulus and the thickness of covering for DLR LWR III without load. The impact speed is $v_{r, 0}=2 \mathrm{~m} / \mathrm{s}$ and the radius of the robot is $R_{r}=0.08 \mathrm{~m}$.

collisions between a human head and a robot with/without covering. With this tool it is possible to design safer robots and determine human-friendly operating conditions under the given safety constraints.

2) Safety criteria for soft-tissue injuries were introduced to estimate skin injury due to blunt head impacts. We could also observe that for the given conditions lacerations are generally accompanied by contusions.

3) In order to prevent skin injuries from blunt impacts the appropriate elastic modulus and thickness of a covering that is attached to a robot can be determined. As this evaluation can be conducted at the design stage of a manipulator, considerable time and cost can be saved.

\section{ACKNOWLEDGMENTS}

This work has been partially funded by the European Commission's Sixth Framework Programme as part of the project VIACTORS under grant no. 231554. This work was supported by the Center for Autonomous Intelligent Manipulation under Human Resources Development Program for Robot Specialists.

\section{REFERENCES}

[1] S. Haddadin, A. Albu-Schäffer, M. Frommberger, J. Roßmann, and G. Hirzinger, "The "DLR crash report": Towards a standard crashtesting protocol for robot safety part I: Results," IEEE International 


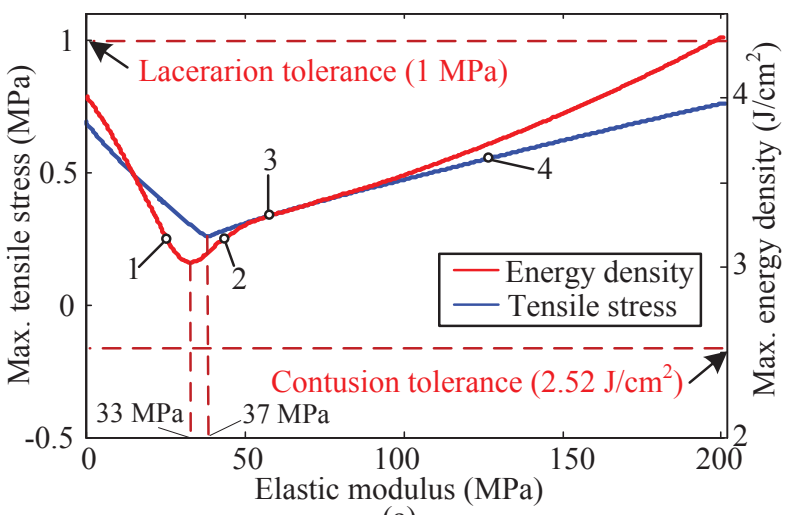

(a)

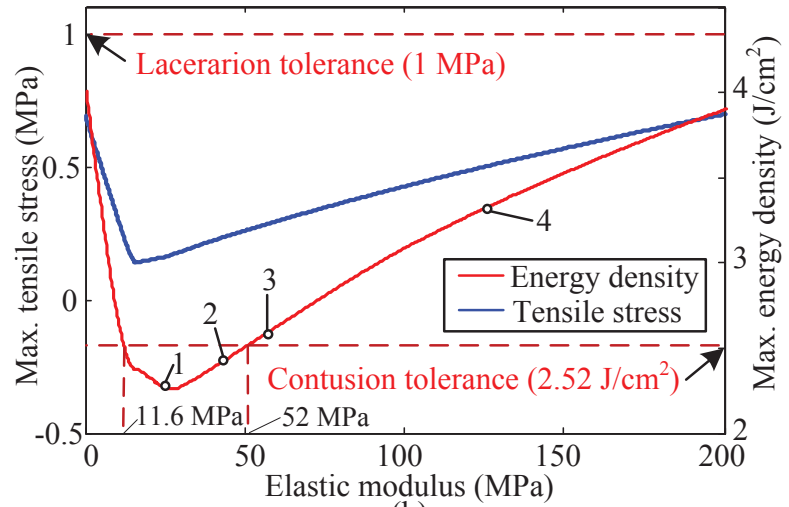

(b)

Fig. 13. Maximum tensile stress as a function of elastic modulus for $b_{c}=10 \mathrm{~mm}$ (upper) and $b_{c}=15 \mathrm{~mm}$ (lower). The impact speed is $v_{r, 0}=2 \mathrm{~m} / \mathrm{s}$ and the radius of the robot is $R_{r}=0.015 \mathrm{~m}$.

Conference on Robotics and Automation (ICRA2009), Kobe, Japan, pp. 272-279, 2009.

[2] S. Haddadin, A. Albu-Schäffer, M. Frommberger, J. Roßmann, and G. Hirzinger, "The "DLR crash report": Towards a standard crashtesting protocol for robot safety part II: Discussions," IEEE International Conference on Robotics and Automation (ICRA2009), Kobe, Japan, pp. 280-287, 2009.

[3] Y. Yamada, Y. Hirasawa, S. Huang, and Y. Umetani, "Fail-safe human/robot contact in the safety space," International Workshop on Robot and Human Communication, pp. 59-64, 1996.

[4] J.-J. Park and J.-B. Song, "Collision analysis and evaluation of collision safety for service robots working in human environments," International Conference on Advanced Robotics, 2009.

[5] J.-J. Park, B.-S. Kim, J.-B. Song, and H.-S. Kim, "Safe link mechanism based on nonlinear stiffness for collision safety," Mechanism and Machine Theory, vol. 43, no. 10, pp. 1332-1348, 2008.

[6] A. Bicchi and G. Tonietti, "Fast and soft arm tactics: Dealing with the safety-performance tradeoff in robot arms design and control," IEEE Robotics and Automation Magazine, vol. 11, no. 2, pp. 22-23, 2004.

[7] R. Schiavi, G. Grioli, S. Sen, and A. Bicchi, "VSA-II: a novel prototype of variable stiffness actuator for safe and performing robots interacting with humans," IEEE International Conference on Robotics and Automation, pp. 2171-2176, 2008.

[8] T. Morita and S. Sugano, "Development and evaluation of seven dof mia arm," IEEE International Conference on Robotics and Automation, pp. 462-467, 1997.

[9] M. Zinn, O. Khatib, B. Roth, and J. K. Salisbury, "A new actuation approach for human-friendly robot design," International Journal of Robotics Research, vol. 23, no. 4/5, pp. 379-398, 2005.

[10] S. Haddadin, A. Albu-Schäffer, and G. Hirzinger, "Requirements for safe robots: Measurements, analysis \& new insights," Int. J. of Robotics Research, vol. 57, no. 11-12, pp. 1507-1527, 2009.

[11] _ , "The role of the robot mass and velocity in physical human-robot interaction - part I: Unconstrained blunt impacts," IEEE International Conference on Robotics and Automation (ICRA2008), Pasadena, USA, pp. 1331-1338, 2008.

[12] interaction part II: Constrained blunt impacts," IEEE International

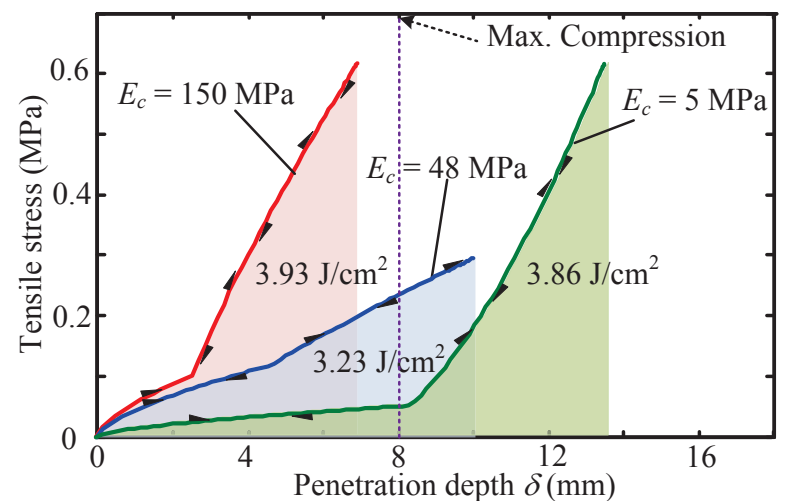

(a)

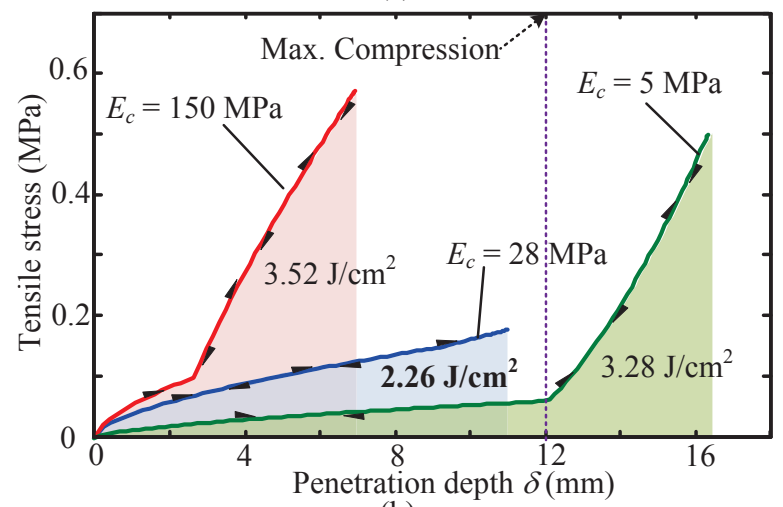

(b)

Fig. 14. Tensile stress/displacement curve of three materials for $b_{c}=$ $0.01 \mathrm{~m}$ (upper) and $b_{c}=0.015 \mathrm{~m}$ (lower), $v_{r 0}=2 \mathrm{~m} / \mathrm{s}$, and $R_{r}=0.015 \mathrm{~m}$.

Conference on Robotics and Automation (ICRA2008), Pasadena, USA pp. 1339-1345, 2008.

[13] M. Wassink and S. Stramigioli, "Towards a novel safety norm for domestic robots," IEEE/RSJ Int. Conf. on Intelligent Robots and Systems (IROS2007), San Diego, USA, pp. 3243-3250, 2007.

[14] S. Haddadin, A. Albu-Schäffer, and G. Hirzinger, "Soft-tissue injury in robotics," IEEE International Conference on Robotics and Automation (ICRA2008), Anchorage, Alaska, pp. 3426-3433, 2010.

[15] K. L. Johson, "Contact mechanics," Cambridge University Press, 1985.

[16] I. P. Herman, "Physics of the Human Body," Springer-Verlag Berlin Heidelberg, 2007.

[17] V. Hodgson and L. Thomas, "Comparison of head acceleration injury indices in cadaver skull fracture," SAE Technical Paper, no. 710854, 1971

[18] V. Hodgson, J. Brinn, L. Thomas, and S. Greenberg, "Fracture behavior of the skull frontal bone against cylindrical surfaces," 14th Stapp Car Crash Conference Society of Automotive Engineers, New York, USA, pp. 341-355, 1970.

[19] D. L. Allsop, C. Warner, M. Wille, and D. Schneider, "Facial impact response - a comparison of the hybrid III dummy and human cadaver," SAE Technical Paper, no. 881719, 1988.

[20] D. L. Allsop, T. R. Perl, and C. Y. Warner, "Force/deflection and fracture characteristics of the temporo-parietal region of the human head," the 35th Stapp Car Crash Conference, pp. 269-278, 1991.

[21] A. M. Nahum and R. Smith, "An experimental model for closed head impact injury," SAE Technical Paper, no. 760825, 1976.

[22] U. Hartmann, "Ein mechanisches Finitite-Elemente Modell des Menschlichen Kopfes," PhD thesis, University of Leipzig, 1999.

[23] O. Khatib, "Inertial properties in robotic manipulation: An object-level framework," International Journal of Robotics Research, vol. 14, no. 1, pp. 19-36, 1995.

[24] S. Haddadin, A. Albu-Schäffer, and G. Hirzinger, "Safe physical human robot interaction: Measurements, analysis and new insights," International Symposium on Robotics Research, pp. 439-450, 2007.

[25] H. Yamada, "Strength of biological materials," The Williams and Wilkins Company, 1970. 